\title{
Los retos de la planificación universitaria en el marco de la crisis del presente: una revisión alternativa
}

\section{The challenges of university planning in the context of the present crisis: an alternative review}

\author{
José Jair González-López \\ Grupo MYSCO \\ Universidad de Ibagué \\ Ibagué, Colombia \\ jair.gonzalez@unibague.edu.co \\ Hernán López-Garay \\ Grupo MYSCO \\ Universidad de Ibagué \\ Ibagué, Colombia \\ hernan.lopez@unibague.edu.co
}

\author{
Marly V. Tafur-Osorio \\ Grupo MYSCO \\ Universidad de Ibagué \\ Ibagué, Colombia \\ marly.tafur@unibague.edu.co
}

\author{
Darío A. Martínez-Leal \\ Grupo MYSCO \\ Universidad de Ibagué \\ Ibagué, Colombia \\ dario.martínez@unibague.edu.co \\ Daniel Lopera-Molano \\ Grupo MYSCO \\ Universidad de Ibagué \\ Ibagué, Colombia \\ daniel.lopera@unibague.edu.co
}

\begin{abstract}
Resumen-Las universidades en su historia han experimentado diversos cambios y se han visto enfrentadas a factores adversos, que siguen vigentes, como: la exclusión, el elitismo y una marcada influencia de las estructuras de poder tales como la Iglesia, el Estado y el Mercado. Bajo la lógica del modelo de mercados, la educación, siendo principalmente un derecho, se fue concibiendo como un servicio, de lo cual se instituyó su función económica, además de la social, haciendo necesaria la adopción de modelos de gestión que enfatizan en la sostenibilidad, la organización burocrática, la planificación estratégica y la implementación de mecanismos, cada vez más estrictos de previsión, control y medición del éxito. De este escenario, derivaron diversas tensiones, como: la competencia entre instituciones, las presiones para responder a las regulaciones del gobierno y la necesidad de garantizar la sostenibilidad social y financiera, entre otras; haciendo de la autonomía y la libertad de cátedra un reto mayúsculo, en un escenario de crisis global a nivel político, social, ambiental y económico.En países como Colombia, este escenario de crisis se ha caracterizado, además, por tensiones socio-culturales entre actores que demandan otros modelos de vida, y por ende de una Educación Superior ajustada a las necesidades de transformación desde los territorios y las cosmovisiones allí situadas, haciendo latente la necesidad de modelos educativos y de planificación coherentes y pertinentes en relación con las realidades del país - Ejemplo de ello es la Universidad Autónoma Indígena Intercultural.La gestión universitaria actual, demanda procesos de concepción y direccionamiento diversos, participativos y situados en los contextos, de los cuales emane la formulación de planes de desarrollos institucionales coherentes y pertinentes. Es así como la planeación es un factor clave, en tanto que desde allí es posible sentí-pensar-actuar los procesos de cambio en contextos marcados por la incertidumbre y la crisis epocal. Este artículo plantea un recorrido crítico-histórico del devenir de las universidades y la planificación institucional, desde el cual se erigen y delinean los retos de las Universidades en el momento presente.
\end{abstract}


Abstract-Universities in their history have undergone various changes and have been faced with adverse factors, which are still in force, such as: exclusion, elitism and a marked influence of power structures such as the Church, the State and the Market. Under the logic of the market model, education, being mainly a right, was conceived as a service, from which its economic function was instituted, in addition to the social one, making it necessary to adopt management models that emphasize sustainability., bureaucratic organization, strategic planning and the implementation of increasingly strict mechanisms for forecasting, controlling and measuring success. From this scenario, various tensions arose, such as: competition between institutions, pressures to respond to government regulations, and the need to guarantee social and financial sustainability, among others; making autonomy and academic freedom a major challenge, in a scenario of global crisis at a political, social, environmental and economic level.In countries like Colombia, this crisis scenario has also been characterized by socio-cultural tensions between actors who demand other models of life, and therefore a Higher Education adjusted to the needs of transformation from the territories and worldviews located there, making latent the need for coherent and pertinent educational and planning models in relation to the realities of the country - An example of this is the Autonomous Intercultural Indigenous University-. The current university management demands processes of conception and direction diverse, participative and situated in the contexts, from which the formulation of coherent and pertinent institutional development plans emanates. This is how planning is a key factor, since from there it is possible to feel-think-act the processes of change in contexts marked by uncertainty and the epochal crisis. This article presents a critical-historical journey of the future of universities and institutional planning, from which the challenges of universities at the present time are erected and outlined..

\section{Keywords- component: college planning, development, epochal crisis, transformation}

\section{Introducción}

La crisis de nuestra época y más recientemente su profundización a raíz de la situación epidemiológica signada por el virus corona, ha generado un acelerado cambio en las formas dominantes de las organizaciones políticas, sociales y culturales, haciendo visible y crítica diferentes problemáticas como la desigualdad y las medidas para detener catástrofe ecológica, también evaporando la sensación de seguridad y estabilidad, abocando procesos necesarios y creativos de reenmarcación de las complejas problemáticas sociales que no dejan lugar a las soluciones tradicionales y rígidas [1]. El contexto de las Instituciones de Educación Superior no se escapa a esta situación, en tanto esta se encuentra atrapada por las fuerzas del mercado, y por tanto sus energías se han abocado a sobrevivir en un entorno de economía apremiante, ante la baja significativa de matrículas, lo que, en su afán por sobrevivir, le ha llevado a redoblar esfuerzos para incrementar la población estudiantil, diversificar la fuente de ingresos $y$ financiación, fortalecer la imagen institucional, competir por una mayor visibilidad en rankings, trabajar para la certificación en calidad y fortalecer la infraestructura tecnológica, entre otros aspectos más, con los que se busca sustentar su vigencia y garantizar su permanencia.

Ante este panorama de creciente presión, las fuerzas del mercado lesionan significativamente su papel en la construcción de la conciencia crítica que reclama una institución que debe servir a la sociedad y ser faro orientador para construir una vida buena para todo el conjunto de la sociedad (no un buen mercado), desde la formación de buenos ciudadanos. Es así como las Universidades, tanto públicas como privadas, se han convertido en cajas de resonancia de las condiciones que producen y re-producen la crisis.

Asistimos, por lo tanto, a un momento crítico en el que la Universidad debe revisar su sentido, su accionar y su ser. Para ello, debe ubicarse como un centro de pensamiento y acción basado en el cuidado de la comunidad interna y externa, la naturaleza y la totalidad de la región como territorio. Para ello se hace necesario fortalecer la universidad, pensando críticamente sus perspectivas de desarrollo y sus procesos de planeación, en tanto que actualmente estos funcionan como un producto de la crisis de nuestra época, y por tanto se encuentran atrapados por el pensamiento instrumental: uso de los seres humanos y la naturaleza como medios para la concentración de capital y la maximización de ganancias, dejando de lado el bien común, la justicia social y una relación equilibrada con la naturaleza. Para acometer esta tarea de manera consciente, proponemos responder una pregunta necesaria en este ejercicio académico: ¿Qué condiciones históricas favorecieron la constitución de la universidad actual?, ¿Qué condiciones favorecieron este diseño? y ¿qué escenarios presentes y futuros están planteando estos diseños?

Desde esta perspectiva, el presente documento pretende dilucidar estas preguntas desde una revisión bibliográfica y con perspectiva histórico-crítica de los procesos de desarrollo universitario, que permita entrever en los hilos del tiempo las transformaciones más representativas de las universidades, para comprender mejor su presente. En este recorrido se abordará el papel de las elites y la incidencia de las estructuras de poder tales como la Iglesia, el Estado y el Mercado. Bajo el dominio de este último, veremos cómo la Educación se volcó hacia una concepción de servicio -como cualquier otro en el mercado-, con exigencias de organización bajo la lógica empresarista en sus procesos de planeación y oferta, que llevan a cuestionar su papel en la responsabilidad con la justicia social y ambiental, sobre todo en países como Colombia en los que persisten grandes brechas sociales que derivan de la histórica exclusión de vastos sectores sociales entre los que sobresalen los indígenas, afrodescendientes y campesinos. Estos segmentos de la sociedad han venido planteando, bajo sus cosmovisiones en conexión con la actualidad del país, rutas educativas que respondan con mayor pertinencia y coherencia en sus 
territorios - ejemplo de ello es la Universidad Autónoma Indígena Intercultural-.

Finalizamos este recorrido, señalando algunos de los derroteros que consideramos relevantes para re-imaginar una universidad más coherente con los principios humanistas que deben abrirse paso en el siglo XXI, basados en el respeto por la diversidad socio-ambiental y ante todo la apuesta por futuros que tengan futuro.

\section{Metodología}

El presente trabajo se ubica en la fase de revisión bibliográfica de una investigación con mayores alcances, que busca el diseño de escenarios futuros del contexto universitario desde la narrativización, la especulación y el autorreflexión. Para el desarrollo de este documento, se trabajó desde la metodología para la revisión bibliográfica y la gestión de información de temas científicos, a través de su estructuración y sistematización [2], la cual consta de tres fases: definición del problema, búsqueda de información, organización de la información y análisis de la información.

En la fase de definición del problema se exploró, conjuntamente con el equipo de investigación, las distintas maneras de entender la crisis de la planificación universitaria. Para ello se trabajó desde el enfoque sistémico [3] el cual ubica, de un lado, las manifestaciones del problema, da sentido a los elementos que se enuncian, discute la forma en cómo ha de ser nombrado el problema, ubica causas y consecuencias y distingue interrelaciones, para plantear lo que se distingue como "situación problemática", la cual solo es posible de construir a partir de una comprensión amplia del contexto y de la escucha de varias voces de actores involucrados.

En este proceso inicial de comprensión, se ubicó la crisis actual (Covid-19) como una pequeña manifestación de una crisis más amplia a la que se hizo referencia como la crisis epocal. Ante este contexto, la pregunta de indagación que guio el proceso general de la investigación fue: ¿Cuál debe ser el rol de las instituciones de educación superior ante los retos del presente?, siendo el reto, aportar a la transición de una sociedad más justa y sostenible, a nivel social y ambiental, y navegar en un contexto de crisis económica que amenaza a las Universidades -tensión económica-. Ante este panorama, también nos preguntamos de manera más puntual y para el caso del presente documento: ¿cómo se fueron gestando las Universidades en occidente?, ¿qué condiciones históricas favorecieron su surgimiento? De allí emergieron algunos puntos clave para la revisión de su evolución: sus inicios estuvieron centrados en el cultivo de ciudadanos que contribuyeron al desarrollo del bien común, y desde allí se transformó a las universidades en el presente llegando a ser mecanismos de producción de profesionales para el mercado que tienen que competir, como otras empresas, para lograr subsistir. Emerge entonces la pregunta: ¿Cuáles han sido los cambios más significativos de ese proceso de transformación?, y ¿qué atributos exhiben estos cambios?

Una vez ubicada la problemática y las preguntas guía, se inició la búsqueda de información bibliográfica, se organizó y clasificó la información en carpetas y hojas de cálculo, permitiendo con ello su revisión, luego de lo cual se construyó un mapa de ideas que facilitó la estructuración de la información desde un orden lógico que permitió además ubicar los aportes de cada autor y documento para dar respuesta a las preguntas formuladas.

Posteriormente se avanzó en el proceso de análisis, con la selección de documentos relevantes para dar respuesta a las preguntas de indagación. Se ubicaron el orden jerárquico los estudios con relación directa y otros de menor importancia y se realizó una estructuración inicial, para dar desarrollo a cada tema y sub-tema. A continuación se presenta una síntesis de los resultados obtenidos en esta fase de la investigación.

\section{Imaginario de la Institución universitaria en el contexto moderno}

A lo largo de la historia, las universidades han pasado por diversas transformaciones. La universidad medieval se caracterizaba por su autonomía y se mostraba en formas muy diferentes en su organización y gestión, desde instituciones de propiedad de las comunidades académicas que la gobernaban y con poca intervención de los estudiantes, como las universidades de Cambridge y Oxford, hasta instituciones gobernadas y pagadas por los estudiantes como la Universidad de Bolonia. Con el tiempo, la autonomía de las universidades y su libertad científica se vio afectada por intereses políticos y religiosos, tal como sucedió en la época de la inquisición. La Universidad moderna, que tiene como modelo la Universidad de Berlín, fue concebida como institución libre de la tutela de la iglesia y el Estado, donde la libertad académica individual no fuera afectada por la intervención externa, como una forma de asegurar su búsqueda de la verdad por la verdad [4].

Para la época, ambas tutelas (Iglesia y Estado), eran dos brazos de un mismo cuerpo que ejercían el poder a todas las instancias vitales de la sociedad, heredado de las sociedades clásicas. En el caso de la educación, la organización de los saberes se orientaba desde las siete artes liberales consideradas desde un incipiente currículo de Trívium y el Quadrivium. Es pertinente entonces subrayar que, desde la fundación de la Academia de Platón hasta las reorientaciones de su discípulo Aristóteles, la educación fue vista desde La República, como un mecanismo de adaptación a los grandes ideales del Estado. Es con este legado al que las universidades de la Alta Edad Media asisten; un legado de exclusión, elitismo y de marcada influencia con las estructuras de poder. Así, se puede decir que la organización universitaria ha estado condicionada por el entorno y las transformaciones propias de cada época histórica; sin embargo, el control frente a relaciones de poder ha sido constante; en tal sentido, las instituciones de educación se fueron adaptando a las consideraciones de la estructura de poder.

En el contexto de la Modernidad, autores como Paul Goodman [5], destaca cómo los procesos de escolarización generan ciertas perversidades en las juventudes que participan en procesos de formación institucional, tales como estilos de comportamiento que 
incentivan formas de vida insostenibles, desprecio a lo que consideran como inferior y, certezas de pensamiento crítico que no colocan en tela de juicio el patrón de vida dominante.

Así, desde los planteamientos de Goodman podríamos concluir con la pregunta ¿Para qué sirve la universidad? Interrogante este que se plantea en criterios de funcionalidad, de idoneidad de la institución para cumplir con la función, de su identidad que le permite distinguirse de otras, de su ser y de cómo planea llevar a cabo su función. Dicho cuestionamiento inspirado en Goodman, supone el cuestionamiento ontológico de las instituciones de educación superior, en tal sentido, estas instituciones no se definen desde marcos funcionales, sino desde los componentes distintivos y fenomenológicos que componen el ser de la institución. Hablar del ser, es hablar de los elementos constitutivos que se enmarcan en la temporalidad, de tal manera que el ser se constituye desde su historiografía, su presente y sus connotaciones de futuridad [6]. En otras palabras, para definir la universidad moderna es necesario considerar el pasado como rememoración que constituye y condiciona, el presente que define el acontecer, y el futuro, el cual, para la investigación es una forma de construir el presente.

Ahora bien, en términos distintivos, la función de la universidad es, a groso modo, la enseñanza (transmisión y apropiación del saber), investigación (construcción del saber y generación de nuevo conocimiento) y extensión (articulación y proyección de saberes a la sociedad). En dicho contexto, Everret Reimer [7], destaca que en estas funciones, las instituciones de educación juegan un papel dañino, sosteniendo que en el mundo dominado por la tecnología -moderno- la universidad garantiza la herencia de los que se benefician de la dominación, con la intención de perpetuar el consumo sin fin, garantía del modelo económico dominante maquillado con ideas de superación de estándares personales, institucionales y nacionales. En tal sentido, en el pasado y el presente, la función de la universidad es, y ha sido, instrumentalizar el saber en concordancia con el poder; quedando con ello un lugar en disputa: El futuro. En el anterior contexto, la competencia -patrón fundamental de la educación moderna- se imbrica en lo económico. Ahora, como bien lo han demostrado algunos sociólogos y psicólogos de la educación (Conf. Infra.), gran parte de nuestro conocimiento se da por fuera de las instituciones de educación, y en ocasiones a pesar de esta. Por lo cual, es menester acotar el proceso en el que las instituciones han monopolizado el "saber". En la conexión entre saber y poder, y en la consolidación de su posible ser, es importante señalar que en el cómo se narra la universidad y como desde la percepción es narrada, y como esta aparente autodefinición y concepción externa, justifica la permanencia de las prácticas que se adjudican a su función.

Si bien la monopolización del "saber" por parte de las instituciones educativas es un elemento central de la educación moderna, este no es el único. Diferentes corrientes provenientes del paradigma crítico de la pedagogía, han denunciado cómo los sistemas de educación superior han sido acríticos a los grandes conflictos sociales, económicos y políticos de la sociedad; sustentados en la presencia de prácticas opresivas y contra-liberatorias de la sociedad. Una vez más estas visiones estaban cargadas de gran contexto del pasado, exceso de presente, pero desvinculadas del futuro.

Las influencias teóricas de estos paradigmas críticos, se encuentran en corrientes neo marxistas como las de Louis Althuser y Nicos Poulantzas; y, en corrientes del posestructuralismo, como Gilles Deleuze y Jacques Derrida. Esta influencia ha llevado a considerar unas instituciones de educación superior que se deben erigir primero como proyecto cultural, con el fin de que genere una red de significaciones alternativas a las lógicas del mercado; y segundo, como proyecto político, que sea capaz de agenciar prácticas alternativas al totalitarismo de la razón.

Así, es clara la propuesta de la pedagogía crítica en subrayar el sentido de la educación en dos direcciones que se entrecruzan: El develamiento de las estructuras de poder, y la agencia de la transformación estructural de la sociedad. Ambas dimensiones (develamiento y transformación), son abordadas de manera distintiva por diferentes autores que comparten dicho paradigma. Giroux [8] se centra en concebir la naturaleza ideológica de la universidad, en ella destaca que la universidad tiene un carácter político que puede reproducir la ideología dominante o, por el contrario, facilitar procesos que generen fisuras a la ideología dominante para que emerja una diametralmente diferente. Por su parte Carr [9], va a destacar que en las instituciones de educación se presentan escenarios de disputa, los cuales no solo se dan en ámbitos considerados trascendentales, como el de la política educativa o política curricular, sino en espacios más íntimos de la realidad educativa, como la intencionalidad política del educador. En la consideración de las características políticas del educador, concuerda Hargreaves [10], al destacar el trabajo colonizador de los docentes, los cuales desde su labor pueden generar "saberes" totalizadores, los cuales anulan otras formas de vida. Finalmente, McLaren \& Farahmandpur [11], muestra la precariedad de los esfuerzos multiculturales en la educación, para contrarrestar los estragos que el neoliberalismo engendra en las instituciones de educación.

Es menester destacar el gran valor de transformación que vincula la teoría crítica. En los años sesenta, en Brasil, se consideró que la alfabetización, desarrollada desde los postulados de Paulo Freire, sería perjudicial para la estabilidad política del país. En dicho contexto, Freire sostiene que la educación históricamente ha desarrollado unos mitos que justifican el orden establecido, como: la libertad, el orden, el derecho a la educación; el mito de que la conciencia crítica es un pecado en contra de Dios; el mito de la propiedad privada como única reafirmación de progreso humano, etc. Allí, la educación debe permitir un acercamiento crítico de las realidades y complejidades del mundo.

Ahora bien, teniendo en cuenta los exámenes y las propuestas realizadas por el paradigma crítico, es importante destacar cómo las instituciones de educación han justificado su labor dentro de la estructura social. Iván Illich [11] subraya cómo estos procesos de justificación institucional en la modernidad se dan en otras instancias de la vida, a saber, la familia es concebida como una 
institución de consumo colectivo, desde el qué compramos y cómo compramos. Algunos ejemplos de cómo la vida en la modernidad gira en torno a las instituciones, por un lado la idea de política está mediada por instituciones, como los partidos políticos; La idea de tranquilidad está mediada por ciertos equipamientos, como la iluminación, y está es ofertada por una institución que presta dicho servicio. La sociedad moderna se olvida que la familia, la política y la educación, no está plenamente contenida en las instituciones. Así, la Modernidad está diseñada para generar profundos lazos de dependencia con las instituciones, o como lo nombra Illich [11]: Las burocracias del bienestar social.

La educación convertida como un estándar de medición del "progreso" de los países, es una de las muestras de que la finalidad de la educación moderna no es solamente el "saber" -dando por sentado que tenga esta última función, guiado por el grado de escolarización, cobertura y desempeño en las pruebas estandarizadas. En esta fenomenología de la institución de educación superior, la palabra universidad en la actualidad ha llevado un vaciamiento de sentido, se piensa la universidad desde mallas curriculares, docentes, discentes, investigaciones; custodia, selección, aprendizaje y esto parte de ideas como: "a los jóvenes les corresponde estar en la universidad", "los jóvenes aprenden en la universidad", "a los jóvenes solo se les puede enseñar en la universidad".

Ahora, partiendo de los postulados de Illich [11], la universidad moderna lleva una ritualización del progreso, en el cual, los egresados son llamados a cumplir un servicio para los sectores económicos hegemónicos del mundo. En tal sentido, la universidad acerca al egresado latinoamericano en el contexto del selecto grupo que progresa en continentes diferentes, mientras que lo aleja de sus conciudadanos no profesionales. En tal sentido, el egresado se adhiere no al "progreso", sino que reproduce los imaginarios de progreso. Así, el nivel de escolaridad también asegura mayor margen de consumo. Esta conexión entre consumo y universidad no es propia de la Modernidad, pues el "saber", desde la educación escolástica en los siglos XI y XII, asegura mayor poder adquisitivo y esto favorece condiciones para el consumo. De tal forma, la planeación en la multiuniversidad moderna ha perdido su capacidad de generar escenarios espontáneos y autónomos, para favorecer la administración de los procesos de enseñanza.

Con el tiempo, y de manera progresiva en varios países, el Estado se fue desligando de la financiación de las universidades, dando lugar a la creación de universidades privadas, además de las ya existentes universidades públicas. La educación pasó de ser un derecho a ser un servicio y los alumnos fueron asumiendo el papel de clientes [4]. Por su parte, el fortalecimiento de los sistemas productivos y la especialización del trabajo, situación que se acentúa con la revolución industrial, motivaron la diversificación de carreras, la profesionalización en la educación, la fragmentación por disciplinas y un mayor énfasis en la formación técnica y el desarrollo de habilidades, descuidando principios claves de la educación como la formación integral y la interdisciplinariedad. A su vez, la globalización de la economía y la transformación del conocimiento en un factor de competitividad, hicieron que además de la función social, las instituciones fueran incorporando la función económica, lo cual influyó en sus modelos de gestión. Lo anterior, sumado al crecimiento de la población, el desarrollo tecnológico, la competencia entre instituciones y la necesidad de optimizar procesos y recursos en procura de la sostenibilidad financiera, entre otros, llevó a que las universidades se fueran transformando en una organización que se preocupa más por su gestión, planificación, previsión, control y éxito, y menos en su propósito de formación, generación de conocimiento y su proyección para el desarrollo de las comunidades.

Del anterior contexto se puede considerar que, hay unas características concretas que han llevado a considerar el ser de la universidad desde criterios funcionales. Estas características parecen confluir en el concepto de saber. Desde la perspectiva crítica, se ha denunciado en características de medios y consecuencias, y en menor medida desde causas, la instrumentalización del saber para generar escenarios de dominación, los cuales devienen en crisis. Sin embargo, estas perspectivas críticas siguen fundamentando, con bastante elocuencia, la función de la universidad como precursora de procesos liberatorios.

Pese a las críticas y el terreno que abona para enmarcar posibilidades de transformación, consideramos que es necesario destacar propuestas de universidades que no solo se fundamenten en la función de las instituciones. Para lo cual, es necesario reconocer algunos escenarios que se erigen en disputa, los cuales permitirán concebir las posibilidades de universidad desde el ser de esta. En virtud de lo anterior, destacamos tres problemáticas identificadas en los párrafos anteriores:

1. Fragmentación de sentido: La organización de las instituciones de educación superior pasa por diseños fragmentados en partes, los cuales no logran articulación -fragmentación de áreas o disciplinas del saber-. Dicha fragmentación es una síntesis de la escisión de la vida; la tragedia de Ícaro y el mito fundacional de occidente, destacan el cómo el hombre para definirse como humano debe de separarse de la naturaleza; así, la universidad para justificar su supuesta función, debe de separarse de la cotidianidad, lo popular, lo mítico, lo subjetivo, de los sentires, entre otros. Por lo tanto, se muestra como imprescindible generar narrativas que logren hacer sentido holístico de la existencia.

2. Exceso de futuridad: Las propuestas de cambio de las instituciones de educación superior, parten de una fórmula que pareciera fuese ley: Se piensa el futuro desde el presente. Dicha fórmula tiene una hipótesis: hay unos líderes en los que se le direcciona toda la expectativa de cambio. Cómo no se ha comprendido el presente, las acciones no siempre perfilan un futuro sustentable. Por lo cual, es necesaria una especulación que ayude a actuar desde el futuro para cambiar el presente, y confiar más en la esperanza que en la expectativa.

3. Las instituciones de educación superior, en la relación sujeto-objeto, siendo esta siempre sujeto y nunca objeto, 
no se ha observado y por ende auto-impugnado. De allí que, la autorreflexión sea un elemento fundamental para develar su ser.

\section{Normativa de la planeación universitaria en Colombia: La ideología pedagógica del Estado}

En la actualidad se preserva parte las ideas fundantes de la universidad moderna, expuesta en los anteriores párrafos, en la medida en que se reconoce la autonomía universitaria a través de principios, que, para el caso colombiano, fueron incorporados en la Constitución del 91 (Artículo 69) y luego en la Ley 30 de 1992 (Artículo 28), y validados por la UNESCO en el 97; las presiones del mercado, la competencia entre instituciones, las regulaciones del gobierno y la necesidad de incrementar y diversificar su fuente de ingresos para garantizar su sostenibilidad; hacen que en la práctica, el concepto de autonomía y libertad de cátedra, esté en riesgo.

Para responder a los retos de eficacia, eficiencia y calidad, que impone la gestión universitaria actual, se ha visto la necesidad de direccionar estratégicamente a las Instituciones a través de la formulación de planes de desarrollo institucional que buscan conducir a las universidades, ante diversos escenarios, hacia el cumplimiento de su misión, objetivos y metas. Este direccionamiento estratégico se vincula con los procesos de acreditación, en cuanto se articula el plan de desarrollo con los planes de mejoramiento que surgen de la autoevaluación, para, de esta forma, asumir los desafíos de la calidad que demanda el sistema universitario y gubernamental. La planeación se ha convertido en una actividad característica de las universidades y se vuelve cada vez más necesaria, dado el alto nivel de cambio e incertidumbre en el ámbito económico, político, social y tecnológico [13].

Dada la complejidad y tamaño de la organización y la gestión universitaria, la planeación se desarrolla en diferentes niveles que podemos denominar: macro, meso y micro, siendo usual la participación de diferentes estamentos de la comunidad, mediante representación en órganos colegiados, mesas de discusión, grupos focales o encuestas, según sea el caso y tipo de organización.

En la planeación de nivel macro, la estrategia se enfoca usualmente hacia el crecimiento y desarrollo de la Institución en torno a las prioridades que se identifican, generalmente, desde la alta dirección. Los planes de desarrollo se diseñan, generalmente, alrededor de grandes estrategias y se estructuran en torno a proyectos que, en parte, buscan satisfacer demandas del mercado: incremento de matrículas, posicionamiento de la imagen, sostenibilidad, diversificación de ingresos, ampliación de cobertura o fortalecimiento de la infraestructura para el desarrollo de sus funciones sustantivas: docencia, investigación y relación con el medio externo. Los métodos de planeación, usualmente adoptados del ámbito empresarial, conducen a la formulación de proyectos en los que se definen objetivos, metas, acciones e indicadores de logro, marcando de esta forma un derrotero en la institución para los siguientes años.

La planeación de nivel meso se enfoca a desarrollar las funciones sustantivas en cada una de las unidades funcionales (facultades, programas, departamentos, centros, unidades), y busca garantizar el fortalecimiento y sostenibilidad de cada unidad o programa, no siempre de manera holística. Esto incluye, entre otros: planeación curricular, planeación del sistema de investigación, planeación de la interacción con el medio externo y planeación de las funciones y recursos de apoyo: presupuesto, finanzas, bienestar y calidad en los procesos, entre otros.

En un nivel micro, se localiza la acción planeada a nivel de programas, proyectos, cursos o similares. Esto incluye el plan de asignaturas, los proyectos de investigación, los proyectos de extensión, los programas de bienestar, entre otros.

Ahora bien, en las consideraciones de las directrices que llevan a que las instituciones de educación superior formulen, promuevan y lleven a la práctica procesos de planeación, se debe enmarcar un análisis de los condicionantes institucionales-jerárquicos, que determinan un deber ser dentro de la organización universitaria.

En primera medida, para el caso colombiano, el Ministerio de Educación Nacional (MEN) es el órgano estatal que orienta, regula y controla los procesos de práctica educativa, en aspectos normativos, pedagógicos y curriculares. En virtud de lo anterior, se destacan dos formas en las cuales el MEN administra el control de los procesos educativos, la primera en criterios facultativos, y la segunda en aspectos vinculantes.

En criterios facultativos, se entenderá la posición de experticia que tiene el MEN. En aspectos formativos, subraya algunas orientaciones pedagógicas y curriculares, las cuales las instituciones de educación superior tienen la libertad de acoger. Esto en criterios institucionales queda claro, sin embargo, en características jerárquicas, se encuentra cierta incongruencia entre la libertad de acción de las instituciones y las políticas educativas. La libertad, se ve condicionada por el concepto de calidad, enmarcado desde lógicas derivadas de directrices internacionales. Así por ejemplo, las pruebas estandarizadas son factores de medición de calidad, de dicha calidad depende que las instituciones de educación superior logren visibilidad, posición y mayor brazo financiero; y es justamente allí, donde las directrices dejan de ser dependientes de la libertad de cada institución. De tal manera, las instituciones constituyen un sentido pedagógico, unos lineamientos curriculares, y unas prácticas didácticas, orientadas por los marcos de calidad establecidos.

En criterios vinculantes, se comprenderá el marco normativo que determina la organización institucional de la educación superior. Acá las disposiciones del MEN deben de ser cumplidas por las instituciones de educación. En esa misma dirección, el MEN adelanta acciones de inspección y vigilancia, preventivas y 
correctivas, para garantizar la adecuada prestación del servicio.

En criterios formales se encuentran aspectos mixtos, es decir, que integra elementos facultativos y normativos. Tal es el caso de la Ley General de Educación de 1994, y la Ley 30 de 1992, esta última como modificación del Decreto 80 de 1980. De lo anterior, se puede destacar la gran influencia de medidas legislativas en la orientación de la doctrina pedagógica del Estado y no de una voluntad de análisis pedagógico. Dicha orientación legislativa, incluye directrices tanto explícitas como implícitas de planeación, calidad, pertinencia, investigación, innovación, cobertura, financiamiento e internacionalización. Lo anterior, se presenta pese a que las mismas voluntades legislativas desde 1992, promulgan la autonomía de las instituciones de educación superior, en concreto desde el Capítulo IV, artículo 28 de la Ley 30, al destacar que las instituciones tienen libertad de modificar sus estatutos y organizar los procesos internos.

Lo anterior, se pierde en detrimento de la idea, hecha norma en la misma Ley 30, de que la educación debe fomentar la calidad en el cumplimiento del principio orientador del Estado. Dicha disputa entre autonomía y principios orientadores, redunda en que la voluntad legislativa es el resumen de lo que el Estado se ha apropiado como suyo, destacando un deber ser normativo frente a las políticas públicas; acá lo político se comprende como la administración de la vida; en el caso que nos concierne es la administración del sentido de la educación [14].

A su vez, el gobierno colombiano no es autónomo, pues se ve cohesionado a dictámenes de la Organización para la Cooperación y el Desarrollo Económico (OCDE) y el Banco Mundial (BM) los cuales evalúan la Educación Superior en categorías como cobertura, calidad, financiación e internacionalización; esto anudado con los esfuerzos de la Organización Mundial del Comercio (OMC) para integrar las directrices educativas a lo que se considera como Sociedad del Conocimiento. En criterios propiamente de planeación, los planes estratégicos de las instituciones de educación superior para el Siglo XXI, fueron enmarcados por la Declaración Mundial de la Conferencia sobre Educación Superior en el Siglo XXI de la UNESCO, desarrollada en París en 1998, en donde se oriento los planes de acción curricular desde los criterios de internacionalización.

En esta dirección, la disputa por la planeación universitaria tiene de telón de fondo la disputa por el sentido de la universidad. El paradigma gubernamental ha sido reemplazado por un paradigma empresarial, por tanto, la agenda educativa como parte de dicho paradigma, también se ve atravesado por aspectos empresariales y transnacionales.

Ahora, en criterios de la Ley General de Educación, el documento rector del ámbito educativo en el país hace alusión en el Título segundo, capítulo 1 artículo 35 en relación con la conformación del Consejo Nacional de Educación Superior (CESU) y la creación de un Departamento Nacional de Planeación. El Título 3, capítulo 1 , artículo 57, dictamina que las universidades deben organizarse como entes universitarios autónomos con régimen especial y vinculados al MEN en lo que refiere a las políticas y la planeación del sector educativo. En la Ley 115 de 1994 artículo 75, destaca cómo el MEN debe servir como factor para la administración y planeación de la educación para la determinación de políticas educativas a nivel nacional y territorial. El Título VIII, titulado como Dirección Administración Inspección y Vigilancia, en el Artículo 148, le atribuye al MEN el deber de formular las políticas que establecen las metas y aprobar los planes de desarrollo a corto, mediano y largo plazo. Para el cumplimiento de lo anterior, el MEN se apoya en la Junta Nacional de Educación, órgano encargado de la planeación y diseño de las políticas educativas del Estado. Lo anterior es evidencia del papel organizativo del Estado con relación a la planeación universitaria.

En relación con los criterios facultativos, en materia de planeación, el MEN trabaja con la Comisión Nacional Intersectorial para el Aseguramiento de la Educación Superior (CONAES), en el que reposa orientaciones vinculadas a los programas académicos con base en la Ley 1188 de 2008, "por el cual se regula el registro calificado de programas de educación superior", y el decreto 1330 de 2019 establece condiciones institucionales y condiciones de programa para el ofrecimiento y desarrollo de programas académicos en el territorio nacional. Las condiciones institucionales se establecen en relación con: Mecanismos de selección y evaluación de estudiantes y profesores, estructura administrativa y académica, cultura de la autoevaluación, programa de Egresados, modelo de bienestar y recursos suficientes para garantizar el cumplimiento de las metas. Las condiciones del programa, se relacionan con: Denominación del programa, justificación del programa, aspectos curriculares, organización de actividades académicas y proceso formativo, investigación, innovación y/o creación artística y cultural, relación con el sector externo, profesores, medios educativos e infraestructura física y tecnológica

La planeación, también se ve condicionada en criterios de evaluación, certificación y acreditación de la institución, en un sentido facultativo. El MEN trabaja de la mano con diversas organizaciones, como la Red de Agencias Nacionales de Acreditación (RANA), de tal manera, se trata de una planeación vinculada a la política de internacionalización de la educación superior que busca contar con reconocimiento internacional. Por su parte, el MEN, a través del Consejo Nacional de Acreditación, ha definido unos lineamientos para la evaluación de alta calidad en instituciones y programas, a través del Acuerdo CESU 02 de 2020, por el cual se actualiza el modelo de acreditación en alta calidad - CNA. Para la acreditación de programas académicos, el CNA establece una serie de características y aspectos alrededor de 12 factores de calidad relacionados con: 1. Proyecto educativo del programa e identidad institucional, 2. Estudiantes, 3. Profesores, 4. Egresados, 5. Aspectos académicos y resultados de aprendizaje, 6. Permanencia y graduación, 7. Interacción con el entorno nacional e internacional, 8. Aportes de la investigación, la 
innovación, el desarrollo tecnológico y la creación, asociados al programa académico, 9. Bienestar de la comunidad académica del programa, 10. Medios educativos y ambientes de aprendizaje, 11. Organización, administración y financiación del programa académico y 12. Recursos físicos y tecnológicos.

En una misma dirección facultativa, se encuentran criterios de planeación influidos por conceptos de innovación y competitividad dentro de tres lógicas: la primera, la regionalización desde los criterios de cerrar brechas desde alianzas rurales; internacionalización y relación Universidad - Empresa - Estado, en un sentido último de adecuar los PEI con las necesidades del mercado laboral. De otro lado, otro concepto que se enmarca como importante es el de Capital Humano, en dicho sentido, la planeación es concebida como la necesidad de cualificar los procesos de formación profesional para abordar procesos de inclusión e interculturalidad, además, de generar competencias y habilidades con el fin de identificar aspectos cualitativos y cuantitativos para las necesidades actuales y futuras de lo que se considera como recurso humano.

Este tipo de condicionantes y determinantes de los procesos de planeación en las instituciones de educación superior, ha sido cuestionado por diferentes estamentos académicos en los cuales se reflexiona no desde los actores legislativos, sino que, desde aspectos esencialmente pedagógicos, así por ejemplo, La Conferencia Regional de Educación Superior en el 2008, sostiene que el sentido de la educación superior no puede quedar regida por reglamentos previstos por el comercio o el mercado, puesto que la educación suministrada por proveedores transnacionales favorece una educación descontextualizada.

De lo anterior, se puede concluir dos cosas: La primera es el grado de injerencia estatal en criterios de la planeación, y segundo, los marcos de sentido que resulta de dicha injerencia. Si bien pareciera que las instituciones de educación superior cuentan con libertad para adelantar su propia planeación y gestión, esta libertad se ve subsumida por el concepto de calidad. Ahora bien, la calidad no es un concepto neutro, puesto que, emerge de relaciones de poder claramente definidas, y no de reflexiones pedagógicas endógenas, así, la calidad viene en estrecha relación con el de internacionalización y estandarización que, de alguna forma, atenta contra la autonomía universitaria.

Por otro lado, en criterios de investigación desde el eje de la planeación, se encuentra un vacío en las indagaciones académicas. Con base en la información recopilada en el 2019 por el grupo de investigación MYSCO de la Universidad de Ibagué, relacionada con las tendencias en investigación educativa en las universidades del país (77 instituciones y 423 grupos de investigación orientados a la investigación educativa), se encontró que para los años 2018 y primer semestre del 2019, había una tendencia en investigar frente a la cultura, la sociedad de la información y política educativa, pero no en particular de la planeación educativa. Sin embargo, se adelantaron proyectos de investigación desde los conceptos de innovación educativa (desde un ámbito de la administración), la gestión curricular (desde la transformación de mallas curriculares y alineación de los programas académicos), y desde la política educativa (desde aspectos de la gestión educativa desde los órganos de toma de decisiones). En conclusión, de los 508 proyectos de investigación desarrollados en los años mencionados, ninguno trabajó de manera explícita desde la planeación institucional o la planeación educativa. Por su parte, entre los proyectos de investigación registrados en Colciencias, hoy Departamento Administrativo de Ciencia, Tecnología e Innovación, durante los años 2010, 2012 y 2014, ninguno se desarrolló desde la planeación educativa.

\section{IES indígenas y afrodescendientes}

En el marco de los países Latinoamericanos, las comunidades indígenas, afrodescendientes y otros actores sociales que habitan los territorios, han venido generando propuestas de Educación Superior interculturales que tienden tanto a la equidad como a la apertura de escenarios en el que ellos tengan cabida desde sus saberes, cosmovisiones, necesidades y demandas históricas políticas, económicas y sociales. Una expresión de ello es la constitución de las Universidades Indígenas, alineadas con el Convenio 169 de la OIT, y que en su ideario se encuentra situar a la educación en un lugar central del proceso de reivindicación política y cultural [15].

Es así que, desde un enfoque basado en la interculturalización de la educación superior y de ir más allá del legado europeo [16] emerge una apuesta coherente con las luchas y modelos de vida basados en las comunidades indígenas. En Latinoamérica un ejemplo de esta emergencia es La Universidad Autónoma Indígena Intercultural en Colombia, fundada en 2003 y avalada por el CRIC desde el enfoque de Sistema Educativo Indígena Propio (SEIP) que solo hasta el 2018 fue reconocida por el Ministerio de Educación -teniendo como antecedentes en el Decreto 1953 del 2014 orientaciones de creación de las instituciones de educación superior indígenas - a través de la Ley 21 y el Decreto 1811 del 2017.

En esta misma línea se encuentra el Centro Amazónico de Formación Indígena CAFI (1989), fundada en una mirada crítica al pasado, comprensión del contexto actual y futuro; De otro lado la Universidad de las Regiones Autónomas de la Costa Caribe Nicaraguense URACCAN (1992) fundada por pueblos indígenas y afrodescendientes, ofertando una formación intercultural integradora que evite la fuga de cerebros (intra-nacional y exterior) y garantice las condiciones de lucha para transformar el racismo institucionalizado, la marginación y exclusión [17, 18].

La naturaleza de estas IES es diversa, y por tanto atiende a propósitos y formas organizativas heterogéneas, Matos [18] señala que un denominador común es su carácter colaborativo intercultural y diálogo de saberes, que responde a la necesidad de lograr una mejor calidad de vida desde la generación de conocimientos y la formación técnica y profesional.

Las historias de estas IES han sido el resultado de procesos históricos de lucha social organizada de pueblos 
indígenas y afro fundamentalmente, dando un lugar central en su creación y funcionamiento, a su visión futura de autonomía, la organización y administración espiritual, el aprendizaje en torno a las problemáticas históricas, planes de vida y propuestas de "Buen Vivir" desde sus formas de ver el mundo y valores ancestrales y culturales, poniendo en cuestión la idea de desarrollo tradicional centrada en el crecimiento, raíz de muchas de las problemáticas ambientales y sociales de la actualidad [19]

Una de las grandes dificultades de estas Universidades ha sido su reconocimiento, financiación y sostenibilidad ante el Estado, cuyos criterios de evaluación no se alineen con los criterios de calidad, pertinencia y relevancia establecidos por sus fundadores, entendiendo que estos parámetros se encuentran centrados en ideas transnacionalizadas $[18,20]$.

Es conclusión, las IES indígenas emergieron en la historia como respuesta a la inequidad, la violencia y otras formas de exclusión que han impedido a muchos pueblos asentados sobre territorios periféricos, generar procesos de transformación basados en sus maneras particulares de entender el mundo y construir presentes que se tejan con sus cosmovisiones. Desde esta perspectiva, las IES tradicionales siguen situadas en procesos monoculturales [18], lo que expresa grandes retos para las Universidades que deseen encarnar una respuesta a la segregación histórica que ha marcado la división de clases y grupos sociales en el país, aportando así a la transición hacia una sociedad más justa, capaz de promover la pluralidad y diversidad en co-existencia desde diversos escenarios.

Esta tarea en mención, ha sido emprendida por diversos académicos e intelectuales a lo largo y ancho del mundo desde las Universidades y otras instituciones sociales y políticas, constituyéndose en ellas visiones críticas en torno a la modernidad y a la colonialidad del saber y el poder, poniendo en tela de juicio el modelo civilizatorio cuya masificación y fortalecimiento ha sido ayudado por las Universidades a través de los modelos científicos y de intervención social, que han de ser transformados a partir de la creación de condiciones para el pluralismo epistemológico; en ello las IES alternativas están aportando significativamente al debate y a la construcción de otras maneras de construir conocimiento en vínculo con los contextos, sus historias, realidades y diversidades, formas más coherentes con los tiempos de cambio que respondan de mejor manera a la crisis epocal.

\section{Conclusiones}

La crisis actual es la manifestación de una crisis más amplia, si bien, la idea original de universidad se sustentó en la autonomía, la libertad de cátedra, la búsqueda de la verdad y el bien común, su accionar no ha estado libre de las luchas de poder. Las universidades partieron de la necesidad de materializar los ideales de la polis y la democracia de la época clásica, posteriormente se sintonizaron con los ideales de un ciudadano cristiano tributario al orden feudal que fue decantando en el poder que adquirieron los mercaderes, de donde se afianzó la relación entre saber y consumo para la acumulación de capital. De este último proceso devino una Universidad aliada con el brazo económico-político de la sociedad, y por tanto tributaria a la idea de progreso y desarrollo que luego se fue fracturando al entender su función social.

A lo largo del documento se construyó una narrativa en la que se evidencia el papel que han tenido las fuerzas del mercado en la constitución de las universidades y, como consecuencia de ello, la preocupación por su supervivencia; situación ésta que ha tomado las riendas de su direccionamiento, entendiendo que cada vez está más amenazada su sostenibilidad financiera, en medio de un ambiente que demanda el posicionamiento de imagen y la formación especializada y profesionalizante, lo cual ha alimentado, entre otros aspectos, la fragmentación, la competencia entre instituciones y una planeación lineal que responde prioritariamente a las demandas del mercado, las políticas gubernamentales del momento y las tendencias cambiantes del entorno; desligando su compromiso ético-político con la sociedad y el ambiente, que demanda cambios profundos y sostenidos en su sentido y hacer. Una respuesta al sin-sentido de la Universidad en el mundo contemporáneo, es la respuesta alterna que han tenido las IES indígenas, afro $\mathrm{y}$ campesinas, quienes desde las necesidades de sus territorios y sus cosmovisiones han planteado modelos educativos coherentes con un proyecto de sociedad en la que todos caben y cuentan.

En este contexto cabe preguntarse, ¿acaso el único camino es seguir reproduciendo el modelo de universidad abocada a formular planes de desarrollo que reproduzcan una y otra vez las bases de una época turbulenta?, ¿debemos reforzar o transformar el modo de vida que nos ha traído al presente? (fraccionamiento, competencia, decadencia), o por el contrario, aprovechar la fuerza desestabilizadora de esta crisis para replantear la idea de universidad, con una conciencia clara del lugar en que estamos, del cómo hemos llegado y por qué hemos llegado hasta aquí, y en todo este escenario una pregunta que conecta la trascendencia con el carácter operativo de las transformaciones ¿Qué papel debe jugar la planeación en el momento actual?

Para empezar a plantear una respuesta viable a esta pregunta, iniciamos con situar la relevancia que cobra la idea de una universidad colectivizada en la que se construye, desde dentro y fuera, el cuidado de la comunidad misma, la comunidad externa, la naturaleza y la totalidad de la región como territorio-unidad. Se hace necesario fortalecerla pensando críticamente sus procesos de planeación, en tanto que, actualmente, estos funcionan como un producto de la crisis de nuestra época, atrapándola en ese mundo dominado fundamentalmente por el pensamiento instrumental: uso de los seres humanos y la naturaleza como medios para la concentración de capital y la maximización de ganancias, dejando de lado el bien común. Estas cuestiones nos llevan a pensar y reflexionar si el destino institucional está signado unilateralmente por la pandemia u otras manifestaciones críticas del presente, o por nuestra capacidad para imaginar, reflexionar y construir colectivamente futuros que tengan futuro. Cuando se re 
imagina la Universidad, la pandemia, en el peor de los casos, es un obstáculo, en el mejor de los casos, es una oportunidad para transformar y seguir viajando a esa imagen y futuro deseado.

En este marco de ideas, el reto del trabajo colectivizado de esa comunidad universitaria es transitar de una planeación instrumental/lineal, cuyas formas de actuación son altamente reactivas y reproducen la crisis [1], a una planeación que posibilite construir nuevos sentidos desde una perspectiva que permita pensar la sociedad en su conjunto como una organización fluida, que permita no solo adaptarse a las nuevas condiciones sino proponerse, desde un cerebro colectivo con capacidades para tomar decisiones estratégicas, imaginar soluciones democráticas fundadas en la participación, la solidaridad y la cooperación, poniendo en cuestión aspectos como el emprendedurismo y la competitividad a toda costa [1]. De otro lado, también se hace imprescindible encontrar herramientas para sentí-pensaractuar en el marco de los complejos fenómenos, condición que abre las posibilidades para encontrar nuevas formas de imaginar y hablar, es decir, tener la capacidad de ir más allá de los mismos lenguajes y enfoques actuales desde los que se piensa y se reproduce la crisis.

Para el planteamiento de este problema se ha desarrollado la metáfora del barco como sistema universitario, el cual navega en un mar embravecido (contexto de la crisis); dicho barco busca como misión fundamental formar a las nuevas generaciones dotadas fundamentalmente de la capacidad de cuidar. En este camino se entiende que la formación también implica la dimensión técnica, no obstante, la capacidad de cuidar es fundamental. Y entonces, la pregunta por la planeación dentro de la metáfora se plantea así: ¿cómo podemos manejar, de mejor manera, este barco en el mar embravecido?, ¿Cómo conducir el barco para que llegue a su lugar objetivo? Y de manera concreta ¿cómo fortalecer los órganos directivos que dirigen el barco hacia su propósito, de tal modo que logren hacer viable el sistema universitario? UI.

Desde la metáfora propuesta, la investigación que da sentido a este artículo se propone responder a la siguiente pregunta: ¿qué capacidades/ metodologías proponemos para generar una transformación en la forma como se está planeando actualmente la Universidad? Este trabajo parte de la necesidad de orientar colectivamente cómo enfrentar la crisis, y dentro de ella resignificar el lugar central que tienen los procesos de planeación, que ante la situación problemática demandan el desarrollo de unas capacidades de todos los cuerpos y miembros de dicha comunidad, teniendo como consecuencia su fortalecimiento para la dirección y planeación.

Es así que el punto de partida es re-imaginar y construir nuevas formas de planear la Universidad, pensando en la necesidad de construir una metodología que fortalezca y desarrolle las capacidades requeridas para ello, introduciendo una apuesta por la innovación en el camino para transformar los procesos de planeación. En este camino se resaltan algunos de los derroteros a transitar en este proceso re-imaginativo:

- Escuchar la realidad e interpretar lo que pasa para comprender mejor el presente. Para este propósito se requiere desarrollar una práctica de autoobservación.

- Imaginar una universidad distinta, una universidad que reconoce que su tiempo ha cambiado. Para ello se requiere empezar a desarrollar capacidades que favorezcan salirnos del marco de ideas convencionales y la toma de conciencia (individual y colectiva) sobre qué significa el modelo actual de vida en el que estamos inmersos; continuar narrativas desde futuros otros (futuros con futuro), estar atento a otras posibilidades y ser capaces de pararse en el futuro para comprender el presente.

- Recuperar una capacidad de narrarnos de forma relacional y especulativa en cuanto que, además de relacionarlos con la vida, la sociedad y sus sentidos, también se narra desde adentro de su propio acontecer y sus posibilidades, dando un constante sentido a lo que hacemos y debemos hacer.

- Transitar a un nuevo orden aprovechando el momento de tensión y crisis que nos tiende la ruptura de la realidad a raíz de lo que se ha denominado pandemia del virus-corona. Para ello será necesario re-significar este evento y comprenderlo en un contexto más amplio a nivel histórico-ontológico.

- Generar nuevos escenarios en los que sea posible dialogar y tender puentes con las fuerzas del mercado, sin dejar que sean estas las que planeen la universidad, priorizando para ello principios fundamentales como el humanismo, la ciencia con conciencia, la pedagogía del cuidado.

- Hacer de la universidad un espacio de la sociedad para conversar, construir e imaginar otras formas de vida que vincule a las comunidades desde una perspectiva de diversidad y pluriculturalidad, que se integren al sistema educativo.

Esto exige formas diferentes de planear, dando cabida al diseño de otros futuros y la toma de decisiones desde una visión sistémica. Exige también una flexibilización en diferentes ámbitos del accionar educativo: flexibilidad curricular, flexibilidad en la gestión y flexibilidad en la interacción con otros actores y con las comunidades.

\section{REFERENCIAS}

[1] B. De Sousa. "La cruel pedagogía del virus." Buenos Aires: CLACSO (2020)

[2] E. Gómez-Luna, et al. "Metodología para la revisión bibliográfica y la gestión de información de temas científicos, a través de su estructuración y sistematización." Dyna 81.184 (2014): 158-163.

[3] E. Aldana Valdés, A. Reyes Alvarado. "Disolver problemas: criterio para formular proyectos sociales". Universidad de los Andes, 
Facultad de Ingeniería, Departamento de Ingeniería Industrial, 2004.

[4] A. Amaral. "Desafíos y perspectivas de la dirección estratégica de las instituciones universitarias. Chile: CNA-Chile, 2009. 15-21.

[5] P. Goodman. Compulsory miseducation. New York: Horizon Press, 1964.

[6] M. Heidegger. "Ser y Tiempo (rustica)." Editorial universitaria, 2005.

[7] E. Reimer. "La escuela ha muerto. Alternativas en materia de educación.” Barcelona: Barral Editores, 1974.

[8] H. Giroux. "Border crossings: Cultural workers and the politics of education. Routledge." 2007.

[9] C. Wilfred. "Una teoría para la educación: hacia una investigación educativa crítica." Ediciones Morata, 1996.

[10] A. Hargreaves. "Profesorado, cultura y postmodernidad: cambian los tiempos, cambia el profesorado.” Ediciones Morata, 1996.

[11] P. McLaren, F. Ramin and N. Cabrera. "La enseñanza contra el capitalismo global y el nuevo Imperialismo: una Pedagogía crítica." Editorial Popular, 2006.

[12] I. Illich. "Obras Reunidas 1 (1.a ed.)." Fondo de Cultura Económica, 2011

[13] P. Torres, Pablo, J. Villafán and. M. Álvarez. "Planeación estratégica y desarrollo organizacional en instituciones educativas: el estudio de un caso universitario en México." Revista Iberoamericana de educación (2008): 2-10.

[14] S, Alzate. "Las políticas públicas en Colombia. Insuficiencias y desafíos". Departamento de Ciencia Política, Universidad Nacional, Sede Medellí, pp. 95-111 FORUM Nro. 1 enero - julio de 2011.

[15] G. Bolaños, L. Tattay and A. Pancho. "Universidad Autónoma, Indígena e Intercultural (UAIIN): Un proceso para fortalecer la educación propia y comunitaria en el marco de la interculturalidad." MATO, Daniel. Instituciones Interculturales de Educación Superior en América Latina. Procesos de construcción, logros, innovaciones y desafíos. Caracas: Instituto Internacional de la UNESCO para la Educación Superior en América Latina y el Caribe (UNESCO-IESALC) 155-190, 2009.

[16] D. Mato, "No hay saber universal, la colaboración intercultural es imprescindible." Alteridades 18.35, 101-116, 2008.

[17] D. Mato. "Las iniciativas de los movimientos indígenas en educación superior: un aporte para la profundización de la democracia." Nueva Sociedad 227/102, 2010.

[18] D. Matos. "Educación Superior y Pueblos Indígenas en América Latina: del "Diálogo de Saberes" a la construcción de "modalidades sostenibles de Colaboración Intercultural". Tramas/Maepova, 4(2), 71-94, 2016.

[19] H. Soto, R. Reinaldo. "i El entretejido de la pluriversidad? Conocimientos en tensión y diálogo en universidades indígenas de Abya Yala: Amawtay Wasi (Ecuador), UAIIN-CRIC (Colombia) y Tupak Katari (Bolivia)." Tesis doctoral, 2019.

[20] D. Mato. "Universidades indígenas en América Latina: experiencias, logros, problemas, conflictos y desafíos." Fundación Equitas; Revista ISEES; 14; 17-45, 12-2014. 